circuit. The standard of frequency at the National Physical Laboratory is a cæsium atomic resonator and this is used to calibrate the quartz clock working standards. These in turn monitor the carrier frequencies of a number of transmitting stations, with the call sign $M S F$, which are operated from the Laboratory from the British Post Office Station at Rugby. Details of these transmissions and of the more powerful carrier wave of the station $G B R$, which is controllod by the $M S F$ standard, are given in the booklet. Separate sections deal with frequency synthesis and measurement and with cavity-resonator frequency meters and their testing and calibration. These meters are most useful in a wide field of applications where an accuracy of about one part in $10^{4}$ is adequate.

The fourth booklet, No. 29, Physical Photometry by Dr. B. H. Crawford, describes those procedures in photoelectric photometry which have their worth in precise measurement and which are in regular use at the National Physical Laboratory. Photometry is essentially the measurement of radiant energy by a selective receiver, the human eye, but individual eyes vary and it is assumed that there is a statistically satisfactory 'standard $\Theta y \Theta$ '. A value for the photopic (light-adapted) standard observer was accepted in 1933 by the International Committee on Weights and Measures and for the scotopic (darkadapted) by the Commission International de l'Éclairage in 1951. These values are tabulated in the booklet. After a general examination of the photoreceptors in present-day use, the spectral correction of vacuum emission photocells and of radiation thermopiles and bolometers is discussed in some detail. Finally, there are two sections dealing with amplifiers and photometers.

S. WEINTROUB

\title{
PETROLEUM CHEMISTRY: U.S.S.R.
}

$\mathrm{T}$ HE past decade has witnessed a spectacular commercial development of the petroleum indus. try in the manufacture and marketing of petroleumderivod organic and inorganic chemicals in Great Britain. Julian M. Leonard, in a recent presidential address to the Institute of Petroleum ( $J$. Inst. Pet., $48,271,1962)$, quotes figures showing that the share of petroleum as raw material for the chemical industry has increased from about 9 per cent in 1949 to 47 per cont in 1959 , at present running at more than 50 per cent and anticipated to reach 65 per cent when 1962 figures aro available. In the United States the production of petrochemicals was a much earlier development than in the United Kingdom and it is estimated that to-day some 80 per cent of organic chemicals manufactured there are based on petroleum. It is doubtful whether the petroleum tochnologist, not directly concerned with modern refinery flowsheets, is himself aware of the phenomenal expansion in this new field and of its technical and economic significance in the future of the world's chemical industry. Certainly the layman is even less informed on this subject, probably because it is not one that can be easily propagated in the popular Press.

The starting point of petrochemicals, as Leonard stated, is the complex of naturally and artificially produced petroleum gases from which are synthesized ethylene, propylene, butadiene, benzene, toluene, xylene, etc. Some of the more important products stemming from these foundations are synthetic rubber, synthetic fibres, detergents, thermoplastics, and agricultural insecticides and fertilizers, many of these roplacing materials formerly derived from purely natural, mainly vegetable resources. The possibility of producing edible oils and fats from petroleum, although not yet matured on a large scale, is none the less practicable and in itself opens up another highly specialized prospect in the chemical industry.

In the academic and technical worlds concerned with petrochemicals there has naturally evolved an extensive literature during the course of development of this fascinating subject, especially in the United States and the United Kingdom. Western European countries have also contributed their share. In these spheres language is no bar to the past or present records of research, discovery or progress. But the same cannot be said of published work in the U.S.S.R., which despite its magnitude and value, must necessarily remain a 'closed book' to all but those chemists who can read the Russian publications per se. The increasing amount of research in the U.S.S.R. on the nature and configuration of petroleum hydrocarbons, composition of sulphur and nitrogen-bearing compounds, and on conversion processes used in the manufacture of petrochemicals, is of such a volume that it has led to the launching in the U.S.S.R. of a new periodical Neftelchimiya-Petroleum Chemistry, which aims at co-ordinating the work of "investigators on the chemistry of petroleum and on the petroloum chemicals industry". This is the quote from the foreword to the English version of Petroleum Chemis. try: U.S.S.R., a quarterly published by Pergamon Press for Pergamon Institute, Oxford, of which the first volume and number* has just appeared in the United Kingdom, under the editorship of D. L. Samuel with translator B. J. Hazzard. The former writes: "Such work is of great interest to all workers in similar or related fields and they will doubtless be well pleased with the opportunitios of reading of the work of their Russian colleagues, made possible by the issue of an English edition. The papers of greatest interest to western research workers are translated in full and an abstract provided of those of lesser appeal".

The first volume of this English publication contains translations of twenty-five original papers by Russian scientists of considerable academic and technical importance in the modern realm of petroleum chemistry in general and petrochemicals in particular. Abstracts of other relevant papers in Neftekhimiya, running to a total of thirty-eight (of which the publishers are prepared to supply full English translations), conclude what, in future numbers, is obviously destined to become a most valuable medium of international exchange of knowledge of petroleum chemistry conceived in the fullest sense of its modern terms of reference.

\section{H. B. MILNER}

- Petroleum Chemistry: U.S.S.R., 1, No. 1; 1962. Publisher guarterly. Translation editor, D. L. Samuel. Pp, 1-218. (London and (85 dollars). 